\title{
Maduración ósea de niños de 6 a 19 años. Muñeca y mano II Parte. Análisis de probitos para huesos aislados
}

\author{
Dr. ('arles V'alenzucla Y.; Dr. Maruricio Canals L.1 \\ Skeletal maturity of children aged 6-19 years. Wrist and hand. \\ Part Two. Probit analysis for isolated bones
}

Th: status quo and probit andysis procedurc uas applied to determine neans and standard deviations of the age a1 which Chilean biys and girls reach the Tanjer - Whitchoulic stages of bone maturation of the wrist and hand. Girls mature carlice as expected. There were mo signiligant difterences with the previous study of the $0-6$ years of age at six years. No deviations from the gaussian distributions wete found.

(Key words: bone maturatom, chilean population, wrist ind hand).

Describimos la maduración ósea de liuesos aislados por el análisis de status quo y probitos en niños de 0 a 6 años." Ahora completamos este estudio con melrachos y muchachas de 6 a 19 años de la misma área de Santiago y estrato socioeconómico.

\section{MUESTRA Y MFTODO}

La mucstra ha sido descriti y corresponde al seguimiento longitudinal rcalizado en el Area Metropolitana Norte desde 1973 cuando los niños ingresaron al primer año de Fnscîanza Básica Fiscal. ${ }^{2}$ Los métodos radiológi$\cos y$ estadísticos han sido descritos en trabajos previos. 1,3,4 No utilizamos en esta ocasión el sistema estadístico SAS y desarrollamos un programa más versátil de probitos. ${ }^{4}$ La nomcnclatura ósea es: $M=$ me tacarpiano; $\mathrm{FP}=$ falange proxima $\mathrm{l} ; \mathrm{FM}=$ falange media; $\mathrm{FD}=$ falange distal; HGR = hue so grande; HGA =hueso ganchoso: $\mathbf{P}=$ piramidal: $S L=$ semiluniar $E=$ escafoides; $\mathrm{TC}=$ trapecio; $\mathbf{T Z D}=$ trapezoide: $P \Gamma=$ pisiforme clasificado en $\mathrm{A}=$ ausente y $\mathrm{B}=$ presente; $\mathrm{SES}=$ sesamoideos que han sido clasificados en $A$ (nínguno), B (uno o más), C (dos o más) y D (cuatro o más). La inclusión del pisiforme y sesamoidecos así como sus clasificaciones Icspectivas son nuestras, no se encuentran en el estudio de Tanner y Whitehouse. El método considera los metacarpianos 1,3 y 5 ; las falanges proximales 1,3 y 5 ; las falanges medias 3 y 5 y las falanges distales 1,3 y 5 . Debido a limitantes de persomal y económicas los jóve-

1. Departamento de Biología Celular y Genética. Facultad de Medicina, Universidad de Chile. nes que no cambian su estatura en tres controles consecutivos son dados de alta: esto deja un sesgo de inmadurez en la muestra que continúa el seguimiento: nuestrat experiencia indica que este sesgo podt ía temer una influencia desde los 16 años en las mujeres y de los 17 en los varones.

\section{RESULTADO}

La tabla presenta los promedios, desviaciones típicas grados de libertad y probabilidades de ajuste al modelo gaussiano de cada hueso con sus estados. Se indica los grados de libertad (G.L.) de la prueba de $\mathrm{X}^{2}$, indicando su probabilidad respectiva; por aproximación decimal la probabilidad mejor que se incluye es 0,01 , aun cuando la probabilidad real fuera inferior a esta cifra.

Las mujeres son más precoces en maduración que los varonęs, $\mathrm{La}$ desviación del modelo gaussiano con probabilidad inferior a 0,05 se produjo en 3 de 105 casos en varones y en 5 de 94 casos en mujeres, lo que está muy ajustado a lo esperado. Hay 35 probabilidades inferiores a 0,5 en 105 casos de varones to que se desvia significativamente de lo esperado $(\$ 2,5)$, es decir, en el caso de los varones la prueba de $\mathrm{X}^{2}$ se desvió significativamente hacia los valores pequeños. En las mujeres hay 46 valores inferiores a 0,5 , lo que no se desvia de lo esperado. ${ }^{47} \mathbf{~ L a}$ modificación del programa de probitos resolvió la mayoría de los problemas del trabajo anterior. 
Tabla

Promedios y desviaciones típicas de edades de maduración ósea método de status Quo y Probitos

\begin{tabular}{|c|c|c|c|c|c|c|c|c|c|}
\hline \multirow[b]{2}{*}{ Hueso } & \multirow[b]{2}{*}{ Est. } & \multicolumn{4}{|c|}{ VARONES $N=673$} & \multicolumn{4}{|c|}{ MUJERES N $=645$} \\
\hline & & $\begin{array}{c}\overline{\mathrm{x}} \\
\text { (mescs) }\end{array}$ & $\begin{array}{l}\text { D.T, } \\
\text { (meses) }\end{array}$ & G.L. & $\mathbf{P}$ & $\begin{array}{c}\overline{\mathbf{x}} \\
\text { (meses) }\end{array}$ & $\begin{array}{l}\text { D.T. } \\
\text { (meses) }\end{array}$ & G.L. & $\mathbf{P}$ \\
\hline \multirow[t]{4}{*}{ Radio } & $\mathrm{F}$ & 67,81 & 12,02 & 3 & 0.77 & 59.87 & 11,79 & 1 & 0,45 \\
\hline & $G$ & $106,9^{\circ}$ & 22,20 & 12 & 0,11 & 104,42 & 16,02 & 8 & 0,68 \\
\hline & $\mathrm{H}$ & 159,06 & 15,01 & 8 & 0,37 & 135,62 & 13,19 & $B$ & 0,84 \\
\hline & 1 & 203,68 & 22,78 & 4 & 0.63 & 177,10 & 14,53 & 6 & 0,87 \\
\hline \multirow[t]{7}{*}{ Cúlito } & B & 89,17 & 15,47 & 5 & 0,17 & 69,42 & 14,88 & 4 & 0,98 \\
\hline & $\mathrm{C}$ & 92,84 & 17,09 & 7 & 0,63 & 75,82 & 15,98 & 5 & 0,37 \\
\hline & $D$ & 107,49 & 18,43 & 9 & 0,74 & 91,35 & 14,52 & 5 & 0,76 \\
\hline & $\mathrm{E}$ & 120,77 & 18,02 & 7 & 0,53 & 103,58 & 12,18 & 5 & 0,30 \\
\hline & $\mathrm{F}$ & 138,05 & 17,31 & 9 & 0,76 & 119,09 & 15,43 & 8 & 0,97 \\
\hline & $\mathrm{G}$ & 163.48 & 14,75 & 5 & 0.57 & 142,47 & 14,45 & 7 & 0,72 \\
\hline & H & 194,56 & 16.70 & 3 & 0.97 & 174,81 & 20.11 & 5 & 0,83 \\
\hline \multirow[t]{6}{*}{ M1 } & $\mathrm{D}$ & 68,39 & 10,69 & 2 & 0.91 & - & - & - & - \\
\hline & i: & 98,87 & 17,98 & 8 & 0,63 & 72,79 & 17,81 & 4 & 0.87 \\
\hline & $F$ & 134,03 & 15,02 & 10 & 0,80 & 108,86 & 12,48 & 5 & 0,55 \\
\hline & $\mathrm{G}$ & 157,86 & 14,69 & 6 & 0,45 & 128,60 & 14,20 & 9 & 0,91 \\
\hline & H & 177,52 & 14,96 & 5 & 0,21 & 152,87 & 14,72 & 6 & 0,42 \\
\hline & 1 & 204,00 & 22,48 & 0 & & 175,98 & 19,04 & 7 & 0,39 \\
\hline \multirow[t]{4}{*}{$M 3$} & $\mathrm{I}^{i}$ & 88,84 & 20,90 & 6 & 0,32 & 81,34 & 15,52 & 3 & 0,91 \\
\hline & G & 146,29 & 16,28 & 10 & 0,76 & 124,23 & 15,89 & 10 & $* 0,01$ \\
\hline & $\mathrm{H}$ & 174,43 & 16,59 & 6 & 0.79 & 149.23 & 15,45 & 7 & 0,40 \\
\hline & 1 & 196,75 & 21,07 & 1 & 0,92 & 176.43 & 17,79 & 6 & 0,24 \\
\hline \multirow[t]{5}{*}{ M5 } & E & 66,19 & 17,65 & 3 & $0,3 \mathbf{I}$ & - & - & - & \\
\hline & $\mathrm{F}^{\mathrm{r}}$ & 120,40 & 19,96 & 11 & 0,95 & 105,68 & 16,97 & 9 & ${ }^{*} 0,02$ \\
\hline & $G$ & 155,56 & 16,34 & 8 & 0,37 & 130,67 & 15,02 & 6 & 0.90 \\
\hline & $H$ & 189,79 & 18,09 & 5 & 0.56 & 161,68 & 14,83 & 8 & 0.25 \\
\hline & I & 196,75 & 21,07 & 1 & $* 0,0 \mathbf{I}$ & 177,01 & 14,99 & 5 & 0.22 \\
\hline \multirow[t]{5}{*}{ l:PI } & I: & 79,21 & 20,31 & 7 & 0,58 & 57,47 & 21,42 & 4 & 0.81 \\
\hline & $\mathrm{F}$ & 130,75 & 17,28 & 8 & 0,43 & 107,01 & 13,74 & 6 & 0,10 \\
\hline & $G$ & 164,00 & 18,05 & 9 & 0,43 & 130,86 & 13.69 & 9 & 0,49 \\
\hline & Il & 192,38 & 19,57 & 5 & 0,85 & 161,58 & 13,38 & 7 & 0,62 \\
\hline & I & 204.00 & 30,32 & o & - & 174,21 & 16,98 & 7 & 0,43 \\
\hline \multirow[t]{4}{*}{$\mathrm{Fl}^{\prime} 3$} & $p^{i}$ & 124,18 & 17,82 & 9 & 0,36 & 100,53 & 16,75 & 8 & ${ }^{*} 0,01$ \\
\hline & $G$ & 158,55 & 14,45 & 7 & 0.75 & 128.24 & 12.96 & 9 & 0,28 \\
\hline & $\mathbf{H}$ & 180,67 & 17,63 & 6 & 0.46 & 152,19 & 14,08 & 6 & 0,37 \\
\hline & I & 197,11 & 21,61 & 0 & - & 172,64 & 15,35 & 7 & 0,49 \\
\hline \multirow[t]{4}{*}{ I:PS } & $\Gamma:$ & 134,06 & 15,18 & 9 & 0,49 & 106.34 & 15.79 & 7 & ${ }^{*} 0,02$ \\
\hline & $G$ & 160,52 & 14,36 & 6 & 0.42 & 129,43 & 12,65 & 8 & 0,58 \\
\hline & $\mathbf{H}$ & 185,84 & 18,61 & 6 & 0,80 & 154.42 & 15,73 & 7 & 0,81 \\
\hline & 1 & 197,11 & 21.61 & 0 & . & 172,44 & 14,67 & 7 & 0,22 \\
\hline
\end{tabular}


Tabla (Cont.)

\begin{tabular}{|c|c|c|c|c|c|c|c|c|c|}
\hline \multirow[b]{2}{*}{ Hueso } & \multirow[b]{2}{*}{ Est. } & \multicolumn{4}{|c|}{ VARONES N $=673$} & \multicolumn{4}{|c|}{ MUJERES N $=645$} \\
\hline & & $\begin{array}{c}\mathrm{x} \\
\text { (meses) }\end{array}$ & $\begin{array}{l}\text { D.T. } \\
\text { (meses) }\end{array}$ & G.L. & $\mathbf{p}$ & $\begin{array}{c}\bar{x} \\
\text { (meses) }\end{array}$ & $\begin{array}{l}\text { D.T. } \\
\text { (meses) }\end{array}$ & G.L. & $\mathbf{P}$ \\
\hline \multirow[t]{5}{*}{ FM3 } & $\mathrm{l}:$ & 74,71 & 20,95 & 6 & 0,51 & 51,09 & 31,01 & 4 & 0,12 \\
\hline & $\mathrm{F}$ & 127.22 & 16,51 & 10 & 0,16 & 107,33 & 1557 & 6 & 0.50 \\
\hline & $G$ & 157.58 & 14,39 & 7 & 0,20 & 127,60 & 14,27 & 9 & 0,90 \\
\hline & II & 189,45 & 15,96 & 4 & 0,36 & 161,55 & 15.73 & 8 & 0,16 \\
\hline & I & 200,18 & 24,80 & 1 & 0,58 & 177,09 & 16,29 & 6 & 0,12 \\
\hline \multirow[t]{6}{*}{ FM5 } & D & 64,99 & 15,33 & 1 & 0.84 & - & - & - & - \\
\hline & $\mathrm{F}$ & 97,32 & 20,90 & 10 & 0,75 & 80,60 & 20,82 & 7 & 0,13 \\
\hline & $\mathrm{F}$ & 142,78 & 15,81 & 8 & 0,35 & 116,67 & 14,13 & 6 & 0,18 \\
\hline & $G$ & 162,73 & 18.27 & 7 & 0,35 & 132,83 & 12,74 & 8 & 0,92 \\
\hline & $\mathrm{H}$ & 190,91 & 16,50 & 3 & 0,61 & 163,12 & 15,67 & 6 & 0,10 \\
\hline & 1 & 196,75 & 21,07 & 1 & 0,92 & 179,56 & 21,23 & 5 & 0,36 \\
\hline \multirow[t]{5}{*}{ FD1 } & $\mathrm{E}$ & 69,44 & 14,72 & 3 & 0,91 & & - & - & -. \\
\hline & $F$ & 104,60 & 20,27 & 10 & 0,64 & 83,58 & 21,02 & 5 & 0,09 \\
\hline & G & 158,18 & 13,23 & 5 & 0,17 & 128,81 & $13,6 ?$ & 9 & 0,91 \\
\hline & H & 180,91 & 14,87 & 7 & 0,16 & 152,46 & 14,00 & 8 & 0,08 \\
\hline & I & 200,10 & 25,00 & 4 & 0.96 & 163,49 & 13,24 & 6 & 0,56 \\
\hline \multirow[t]{5}{*}{ FD3 } & $E$ & 73,84 & 14,50 & 5 & 0,59 & - & - & - & - \\
\hline & $\mathrm{F}$ & 97,91 & 16,15 & 7 & 0,46 & 79,21 & 18,03 & 7 & $0,2 \mathrm{~L}$ \\
\hline & G & 157,07 & 13,14 & 7 & 0,78 & 130,23 & 14,15 & 8 & 0,73 \\
\hline & $\mathrm{H}$ & 183,82 & 14,48 & 7 & 0.69 & 157,29 & 13,93 & 7 & 0,18 \\
\hline & I & 195,02 & 17,16 & 3 & 0,84 & 163,49 & 12,72 & 6 & 0,52 \\
\hline \multirow[t]{5}{*}{ FD5 } & $E$ & 82,39 & 13,13 & 5 & 0,26 & - & - & - & - \\
\hline & $\mathrm{F}$ & 107,97 & 15,99 & 9 & 0,99 & 88,52 & 15,96 & 8 & 0,72 \\
\hline & G & 161,34 & 15,51 & 7 & 0,70 . & 132,42 & 14,63 & $B$ & 0,90 \\
\hline & H & 186,66 & 15,89 & 7 & 0,43 & 157,80 & $14,9 \mathrm{l}$ & 8 & 0,26 \\
\hline & 1 & 198,27 & 19,53 & 3 & 0,92 & 165,02 & 13,72 & 7 & 0,87 \\
\hline \multirow[t]{3}{*}{ HGR } & $\mathrm{F}$ & 65,73 & 16,15 & 3 & 0,74 & - & - & - & - \\
\hline & G & 101,51 & 17,34 & 8 & 0,88 & 86,71 & 14,96 & 8 & 0,53 \\
\hline & $\mathrm{H}$ & 149.57 & 20,23 & 10 & 0,57 & 127,87 & 17,33 & 11 & 0,11 \\
\hline \multirow[t]{4}{*}{$\mathrm{HGA}$} & $F$ & 81,98 & 13,03 & 5 & $* 0,01$ & 71,25 & 14,98 & 4 & 0,98 \\
\hline & $\mathbf{G}$ & 116,07 & 16,13 & 10 & 0,97 & 96,82 & 12,17 & 5 & 0,52 \\
\hline & $\mathbf{H}$ & 145,89 & 17,01 & 9 & $0, ? 1$ & 118,46 & 13,87 & 8 & 0,73 \\
\hline & 1 & 164,02 & 18,16 & 6 & 0,47 & 139,25 & 20,49 & 7 & 0,74 \\
\hline \multirow[t]{5}{*}{$\mathbf{P}$} & D & 52,52 & 27,66 & 5 & 0,61 & - & - & - & - \\
\hline & $\mathrm{E}$ & 84,09 & 19,67 & 7 & 0,60 & 48,30 & 33,07 & 5 & 0,80 \\
\hline & $\mathbf{F}$ & 114,71 & 17,39 & 11 & 0,80 & 98,71 & 14,74 & 8 & 0,44 \\
\hline & $G$ & 139,42 & 17,80 & 10 & 0,91 & 114,89 & 14,58 & 9 & 0,98 \\
\hline & $\mathrm{H}$ & 162,09 & 21,42 & 6 & 0,37 & 134,32 & 17,24 & 10 & 0,37 \\
\hline \multirow[t]{6}{*}{ SL } & C & 58,52 & 27,66 & 5 & 0,61 &. & - & - & - \\
\hline & D & 72,56 & 25,91 & 6 & 0,58 & 58,73 & 19,83 & 4 & 0,90 \\
\hline & $\mathbf{E}$ & 98,36 & 22,04 & 7 & 0,92 & 83,39 & 18,61 & 6 & 0,73 \\
\hline & $\mathbf{F}$ & 124,80 & 19,49 & 12 & 0,72 & 106,16 & 15,07 & 7 & 0,27 \\
\hline & $\mathrm{G}$ & 146,62 & 18,34 & 9 & 0,80 & 125,17 & 13,61 & 9 & 0,23 \\
\hline & $\mathbf{H}$ & 168,81 & 19,21 & 6 & 0,24 & 149,58 & 17,35 & 9 & 0,71 \\
\hline
\end{tabular}


Tabla (Cont.)

\begin{tabular}{|c|c|c|c|c|c|c|c|c|c|}
\hline \multirow[b]{2}{*}{ Hueso } & \multirow[b]{2}{*}{ Est. } & \multicolumn{4}{|c|}{ VARONES N $=673$} & \multicolumn{4}{|c|}{ MUJERES N $=645$} \\
\hline & & $\begin{array}{c}\bar{x} \\
\text { (mescs) }\end{array}$ & $\begin{array}{l}\text { D.T. } \\
\text { (meses) }\end{array}$ & G.L. & $\mathbf{P}$ & $\begin{array}{c}\bar{x} \\
\text { (meses) }\end{array}$ & $\begin{array}{l}\text { D.T. } \\
\text { (meses) }\end{array}$ & G.L. & $\mathbf{P}$ \\
\hline \multirow[t]{7}{*}{ E } & $\mathrm{B}$ & 71,67 & 21,50 & 5 & 0,93 & - & - & - & - \\
\hline & $\mathrm{C}$ & 78,68 & 18,84 & 7 & 0,61 & - & - & - & - \\
\hline & $\mathrm{D}$ & 89,68 & 18,11 & 8 & 0,96 & 65,69 & 16,72 & 5 & 0,69 \\
\hline & $\mathbf{E}$ & 104,69 & 21,32 & 10 & 0,61 & 82,36 & 18,05 & 6 & 0,06 \\
\hline & $\mathrm{F}$ & 124,38 & 18,51 & 10 & 0,73 & 101,05 & 16,58 & 7 & 0,91 \\
\hline & $\mathbf{G}$ & 145,41 & 15,79 & 8 & 0,83 & 120,48 & 13,95 & 8 & 0,58 \\
\hline & $\mathbf{H}$ & 172,20 & 17,20 & 6 & 0,78 & 152,40 & 22,36 & 8 & 0,78 \\
\hline \multirow[t]{8}{*}{ TC } & $\mathrm{B}$ & 65,88 & 29,97 & 6 & 0,95 & - & - & - & .. \\
\hline & $\mathrm{C}$ & 80,00 & 24,77 & 8 & 0,93 & - & - & - & - \\
\hline & $\mathrm{D}$ & 88,66 & 25,03 & 8 & 0,39 & 48,95 & 28,39 & 4 & 0,75 \\
\hline & $\stackrel{E}{ }$ & 106,15 & 21,06 & 8 & 0,50 & 80,19 & 17,03 & 7 & 0,85 \\
\hline & $\mathrm{F}$ & 123,85 & 17,16 & 8 & 0,64 & 97,23 & 14,25 & 7 & 0,27 \\
\hline & G & 144,41 & 13,90 & 8 & 0,71 & 118,40 & 13,11 & 7 & 0,72 \\
\hline & If & 160,90 & 14,49 & 5 & 0,40 & 132,65 & 12,57 & 7 & 0,63 \\
\hline & $\mathrm{I}$ & 179,94 & 20,61 & 8 & 0,93 & 159,13 & 19,16 & 9 & 0,99 \\
\hline \multirow[t]{7}{*}{ T7.D } & B & 75,78 & 17,49 & 5 & 0,91 &.- & - & - & - \\
\hline & $\mathrm{C}$ & 81,21 & 16,98 & 6 & 0,64 & - & - & - & - \\
\hline & D & 89,01 & 17,95 & 6 & 0,71 & 65,30 & 15,74 & 4 & 0,94 \\
\hline & E & 101,38 & 16,46 & 7 & 0,99 & 78,34 & 15,70 & 5 & 0,47 \\
\hline & $\mathbf{F}$ & 120,92 & 16,36 & 7 & 0,61 & 99,08 & 16,26 & 6 & 0,58 \\
\hline & $G$ & 142,70 & 16,73 & 10 & 0,75 & 120,03 & 14,84 & 8 & 0,13 \\
\hline & $\mathrm{H}$ & 159,89 & 18,20 & 10 & 0,89 & 140,27 & 19,19 & 10 & 0,96 \\
\hline $\mathrm{PF}$ & $B$ & 139,44 & $20,7 ?$ & 13 & $* 0,02$ & 107,39 & 17,38 & 9 & ${ }^{*} 0,04$ \\
\hline \multirow[t]{3}{*}{ SI:S } & $B$ & 158,63 & 12.99 & 4 & 0,25 & 129,84 & 14.51 & 8 & 0,11 \\
\hline & C. & 168,64 & 18,28 & 7 & 0,40 & 145,73 & 20,62 & 8 & 0,40 \\
\hline & 1) & 204,70 & 29,75 & 8 & 0,92 & 182,46 & 41,07 & 11 & 0,49 \\
\hline
\end{tabular}

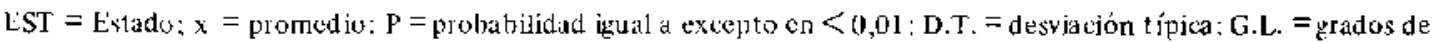
libirtad.

\section{DISCUSION}

No tenemos una explicación para la distorsión asimétrica de la prueba de $\mathrm{X}^{2}$ que ocurrió sólo en varones. La frecuencia extraordinariamente clcvada de valores espcrados muy próximos a los observados, encontrada en el trabajo previo, no se dió en éste, al parecer por las modificaciones que introdujimos al elaborar de nuevo uл programa de probitos. Las mujeres madman antes que los varones como se esperaba. Es probable que el estado superior de maduración de cada hueso se alcance antes y oon una desviación típica menor que la descrita por nosotros debido a que esta
mLiestra está erriquecida con jóvenes que aún están en crecimiento.

\section{RESUMEN}

El método del status quo y análisis de probito se ut ilizó para obtener los promedios y desviacio. nes típicas de la edad a la cual muchachos y muclachas chilenas alcanzan kos estados madurativos óseos de Tannet. Whitehouse de la mano y mujeca. Las mujeres son más precoces según lo esperado. No hubo diferencias significativas con cl estudio anterior de $0-6$ años a los 6 años de 
edad. La muestra se ajustó bien al modelo gaussiano.

\section{REFERENCIAS}

I. I'otenzutela C: $r$.. Canals M. Fergara P.: Maduración óxes de niñus de 0 a 6 años. Muñéa $y^{\prime}$ mano. If Parke, Análisis de probitos para huewos aiskados. Rev (hit Pediatr 1985; 56: 329-333.
2. Avendaño A. Valenzuela C.. Figueroa $L, y$ Coks. Vistudio longitudinal del crecimiento $y$ desarrollo de un $10 \%$ de los miños yue ingresin a la Finseñanza Básica Iriscal. Area Hospitalaria Norte de Santiagro. Pediatría (Santiago) ] 976: 1.9: 1.56-159.

3. Canols M. Valonzuela C.Y., Vergara P.: Maduatación ósea de niños de 0 a 6 años. Muñcea y mano. I Parte. Análisis cuantitiltivo. Rev Chil Pediatr 1985:56: $325-328$.

4. Frney' D.J.: Probit Analysis. Second Ldition. Cambridge at the I niver sity Ptess. 1952. 